\title{
Nanoscale Imaging and Control of Hexagonal Boron Nitride Single Photon Emitters by a Resonant Nanoantenna
}

\author{
Nicola Palombo Blascetta, Matz Liebel, Xiaobo Lu, Takashi Taniguchi, Kenji Watanabe, \\ Dmitri K. Efetov, and Niek F. van Hulst*
}

Cite This: Nano Lett. 2020, 20, 1992-1999

Read Online

\section{ACCESS \\ 山ll Metrics \& More \\ Article Recommendations \\ Supporting Information}

ABSTRACT: Defect centers in two-dimensional hexagonal boron nitride $(\mathrm{hBN})$ are drawing attention as single-photon emitters with high photostability at room temperature. With their ultrahigh photon-stability, hBN single-photon emitters are promising for new applications in quantum technologies and for 2D-material based optoelectronics. Here, we control the emission rate of hBNdefects by coupling to resonant plasmonic nanocavities. By deterministic control of the antenna, we acquire high-resolution emission maps of the single hBN-defects. Using time-gating, we can discriminate the $\mathrm{hBN}$-defect emission from the antenna luminescence. We observe sharp dips (40 nm fwhm) in emission,

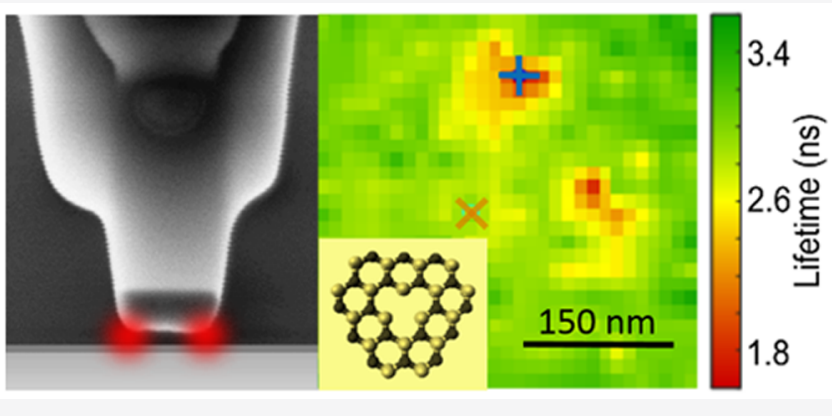
together with a reduction in luminescence lifetime. Comparing with finite-difference time-domain simulations, we conclude that both radiative and nonradiative rates are enhanced, effectively reducing the quantum efficiency. Also, the large refractive index of hBN largely screens off the local antenna field enhancement. Finally, based on the insight gained we propose a close-contact design for an order of magnitude brighter hBN single-photon emission.

KEYWORDS: hBN-defect-center, single-photon-source, nanoantenna, scanning-probe, super-resolution, luminescence-lifetime

$\mathrm{B}$ right and stable single-photon sources are highly ondemand for emerging quantum technologies, such as quantum computing $^{1}$ or encryption schemes, ${ }^{2}$ where nonclassical light sources are required, to provide on-demand indistinguishable photons, one at a time. To this end, quantum dots (QD), ${ }^{3}$ organic molecules in crystalline matrices, ${ }^{4,5}$ diamond nitrogen and silicon vacancy centers, ${ }^{6,7}$ and twodimensional (2D) transition metal dichalcogenides (TMDC) ${ }^{8}$ are all actively explored. Recent progress in efficient coupling of single molecules, ${ }^{5} \mathrm{NV}$ centers, ${ }^{9}$ or $\mathrm{QDs}^{10}$ to waveguides is paving the way to top-down fabricated single-photon sources on-chip.

However, the applications of single-photon emitters face intrinsic limits. The achievable brightness is limited by the luminescence lifetime and intermediate dark states. Molecules tend to bleach, while both molecules and QDs tend to blink. The blinking and bleaching can be alleviated by embedding the molecules in a suitable crystalline matrix ${ }^{11}$ or encapsulating the QDs in an engineered higher bandgap shell. Notably, the brightness can be boosted by enhancing the radiative rate (reducing the lifetime) with an engineered plasmonic antenna at nanometer distance. ${ }^{12,13}$ Unfortunately, the necessary thickness of the protecting shell for photostability, such as the crystalline matrix around molecules or the diamond surrounding the $\mathrm{NV}$ center, does prevent the close proximity of plasmonic antennas.

Recently, the hexagonal boron nitride (hBN) atomic defect is attracting major attention as an alternative candidate for a high-performance single-photon source. ${ }^{14,15}$ The hBN defects are reported to be photostable and free of blinking and bleaching even at elevated operation temperatures as high as $800 \mathrm{~K}^{14,16}$ Most importantly, the emitter is stable in an hBNmonolayer of only nanometer thickness and readily accessible for local nanoscale control and top-down fabrication. Despite the key advantages of photostability and accessibility, the hBNdefect is still limited in brightness as a single-photon source due to its intrinsic luminescence lifetime of several nanoseconds. The atomically thin hBN seems ideal for local enhancement by a plasmonic nanoantenna. To this end, one needs to optimize the coupling between the hBN-emitter and the nanoantenna by deterministic control of position, distance, orientation, and resonance. A few attempts have been reported: Tran et al. relied on nanoantenna arrays ${ }^{17}$ and

Received: December 23, 2019

Revised: February 12, 2020

Published: February 13, 2020 
Nguyen et al. used AFM to couple individual hBN-defects to gold nanospheres. ${ }^{18}$ Both studies reported a slight decrease in radiative lifetime alongside a minor increase in absolute photon emission rates; however, in both cases the crucial emitterpositioning accuracy was a limiting factor. ${ }^{18,17}$ Additionally, the experiments were performed in static systems with fixed positions complicating the systematic study of emitternanostructure interactions.

Here we present a systematic spatially resolved study of the coupling of hBN-defect single-photon emitters to resonant optical nanoantennas with nanometric position-control and optical resolution of $40 \mathrm{~nm}$. By nanosecond time-gating, we separate the hBN-defect emission to record lifetime interaction maps and quantify the interaction strength between emitter and antenna. On the basis of our findings, we propose a nanoantenna-hBN-defect design with an order-of-magnitude enhanced brightness suitable for on-chip top-down fabricated configuration.

First, we analyzed the general optical properties of hBNdefect emitters (Figure 1). Thin $(\sim 5 \mathrm{~nm}) \mathrm{hBN}$-flakes are exfoliated onto a quartz substrate. These pristine flakes show a relatively low defect-density of less than one luminescent site per $50 \times 50 \mu \mathrm{m}^{2}$, moreover mainly located along the flakes' edges. As these areas are generally not well suited for antenna probe scanning, we opt for argon plasma etching, followed by high-temperature annealing, to induce optically stable hBN-

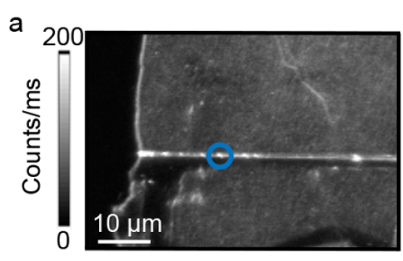

C
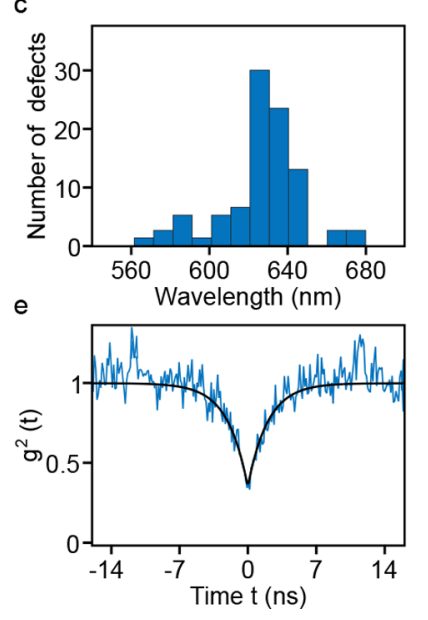

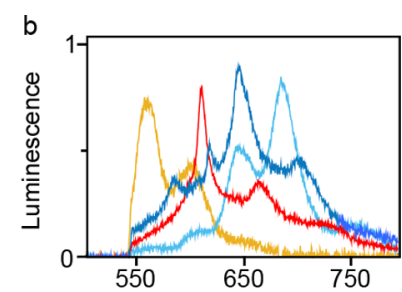$$
\text { d }
$$

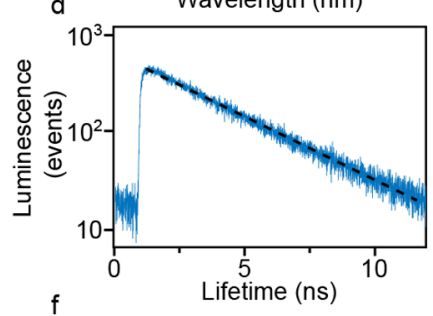

f

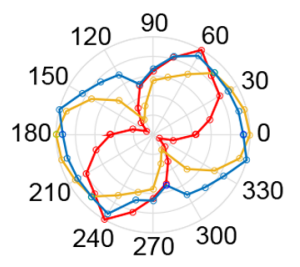

Figure 1. Optical properties of hBN-defect emitters. (a) Confocal luminescence image of an exfoliated hBN flake. The majority of the emitters is located at the edges of the flake, whereas a few oxygenplasma induced defects are present on the flake area itself. (b) Representative emission spectra of various hBN-defects. (c) Histogram of the spectral emission maxima of a total of $95 \mathrm{hBN}$ defects. (d) Typical luminescence decay (blue) and single-exponential fit (red, dashed) with a lifetime of 3.5 ns. (e) Second-order-correlation time response (blue) of the defect highlighted in (a) showing antibunching and exponential fit with 3.5 ns lifetime (red). (f) Representative excitation polarization dependence of three different $\mathrm{hBN}$ emission sites with degrees of polarization of $0.1,0.67$, and 0.9 . defects across the entire flake. ${ }^{19}$ A typical luminescence image of a treated hBN-flake is shown in Figure la. We observe a near-constant luminescence background of approximately 30 $\mathrm{kcnts} / \mathrm{s}$ across the entire flake, diffraction-limited emission sites distributed over the entire flake and pronounced emission along the flake's edges. While the former are mainly induced during plasma etching, the latter are predominantly native defects. The emission spectra of individual defects (Figure 1b) show large heterogeneity, covering a spectral range from 550 to $700 \mathrm{~nm}$ with the mean emission wavelength for plasmaetching-induced luminescent sites centered around $620 \mathrm{~nm}$ (Figure 1c), which is in agreement with previous reports. ${ }^{20,21}$ The photoluminescence decay of individual defects shows a single exponential decay of $\tau=3.5 \mathrm{~ns}$ (Figure 1d), which is in line with previously reported values of a few nanoseconds. ${ }^{14,15}$ To verify the single-photon-emitter nature of the hBN-defects, we record the second-order autocorrelation function. Around $5 \%$ of the measured hBN-defects does exhibit a dip below 0.5 at zero time delay, confirming single-photon emission, whereas the majority of the defects do not exhibit single-photon emission character. ${ }^{19}$ A typical antibunching curve is shown in Figure 1e, exhibiting a $g^{(2)}(0)$ value of 0.4 . This relatively high $\mathrm{g}^{(2)}(0)$ value is because roughly $20 \%$ of the collected photons comes from bulk emission of the hBN-flake. Finally, we determine the dipole orientation of the emission sites by recording the luminescence response on the orientation of incident polarization (Figure 1f). The observed degree of polarization (DOP), computed as $\left(I_{\max }-I_{\min }\right) /\left(I_{\max }+I_{\min }\right)$, varies broadly from 0.1 to 0.9 , without any preferred orientation with respect to the hBN-lattice. ${ }^{22}$ The occurrence of near-isotropic excitation probabilities agrees with the low fraction of single-photon emitting-defects, suggesting the presence of multiple emitters inside a typical $300 \mathrm{~nm}$ confocal spot of our microscope. The density of closely packed hBNdefects probably depends on the plasma etching conditions, used to increase the number of emitters in the exfoliated hBNflakes (SI Methods).

Next, we turn to controlled nanoscale coupling of the hBNdefect emitter to a nanoantenna. To this end, we employ a scanning probe microscope (SI Methods), which allows one to scan a nanofabricated antenna over a single-photon emitter, at a constant distance using shear-force distance control. ${ }^{21}$ Figure 2a,b shows SEM images of the aluminum nanoantenna probes employed in this study, fabricated onto the apex of a heatpulled optical fiber by focused-ion-beam milling (SI Methods). ${ }^{13,23}$ A $160 \mathrm{~nm}$ length of the $50 \times 50 \mathrm{~nm}$ crosssection $\mathrm{Al}$ antenna is fabricated to be resonant with excitation or hBN-emission.

The Al nanoantenna does show weak single-photon luminescence. Therefore, we compared representative hBNdefect luminescence to the antenna signal. Typically a fluence of $40 \mathrm{~kW} / \mathrm{cm}^{2}$ is used, which is one order-of-magnitude below the fluences typically used to excite hBN-defects, ${ }^{14,18}$ and it prevents thermal damage of the nanoantenna. Figure $2 c, d$ shows confocal luminescence images of an isolated $\mathrm{hBN}$-defect as well as the dipolar Al-nanoantenna, excited with circular polarization, while the antenna is kept about $20 \mathrm{~nm}$ from a clean quartz cover glass. Surprisingly, the weak antennaemission is still two times brighter than the hBN-defect. In fact, the hBN-emission is very weak, which represents a considerable challenge for the antenna coupling experiments, as the respective signals have to be separated. An obvious solution would be to employ spectrally selective detection; 

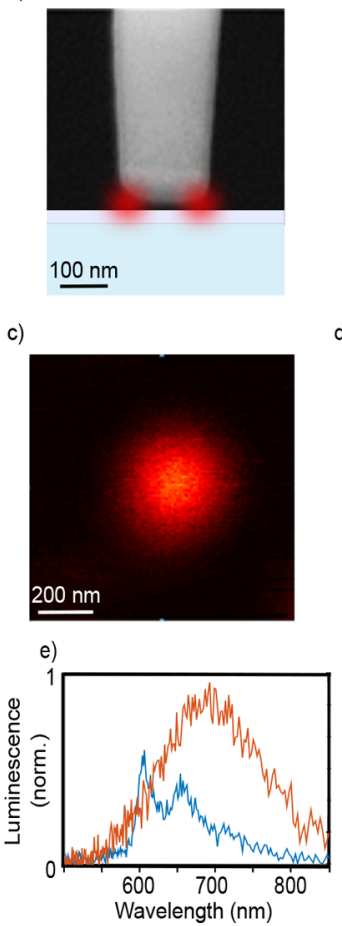

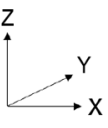

Antenna

hBN

Glass

d)
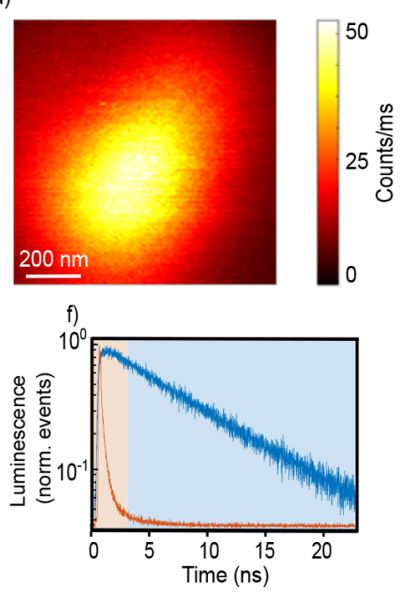

Figure 2. Luminescence of hBN-defect and nanoantenna. (a,b) SEM images of an aluminum nanoantenna probe, either a flat (a) or a $30^{\circ}$ tilted (b) rod antenna, supported on glass. The dipolar antenna has hotspots on either side (red), which couple to the hBN-defect (blue). (c) Confocal luminescence image of a hBN-defect and (d) of the dipolar antenna shown in (a), positioned in close proximity of a clean quartz cover glass, both plotted on the same intensity-scale. (e) Luminescence spectra on same scale of both hBN-defect (blue) and antenna (orange). (f) Photon-arrival histograms for the hBN defect (blue) and the nanoantenna emission (orange).

however, the respective emission spectra exhibit considerable overlap (Figure 2e). Moreover, the intrinsic hBN-emissionheterogeneity (Figure $1 \mathrm{~b}, \mathrm{c}$ ) complicates spectral filtering for enhancing the signal-contrast as different detection filters would be necessary for each defect.

As an alternative to distinguish $\mathrm{hBN}$ and antenna, we employ time-gating of the collected luminescence signal. A comparison of the luminescence time-traces (Figure 2f) shows a rapid decay with $<200$ ps lifetime of the antenna luminescence. After $2 \mathrm{~ns}$, the antenna-signal has essentially decayed to zero, whereas the $\mathrm{hBN}$-signal has gone down only half. Thus, time-gating allows hBN-defect specific detection in a postprocessing manner using time-correlated single-photoncounting detection (SI Methods).

After characterization of $\mathrm{hBN}$ and antenna emission, we now turn toward antenna scanning experiments to acquire interaction maps on the nanoscale. Figure $3 a$ indicates schematically the experiment. First, we position the hBNdefect of interest in the center of our confocal excitation spot. Once positioned, we raster-scan the antenna probe in close proximity across the emission site, while keeping illumination and sample geometry constant, and detect the photonemission as a function of the antenna position.

Figure $3 \mathrm{~b}$ displays a near-field luminescence map with the antenna probe in close proximity to the $\mathrm{hBN}$-defect. Here, we use the flat-dipolar $\mathrm{Al}$ nanoantenna (Figure 2a) fabricated with $160 \mathrm{~nm}$ length for spectral resonance at $620 \mathrm{~nm}$, that is, resonant with the excitation laser. Figure $3 \mathrm{~b}$ shows a bright spot, approximately $300 \mathrm{~nm}$ in diameter, with two darker spots superimposed, slightly offset to the top right of the image, due to nonperfect alignment of the hBN-defect within the confocal illumination spot. The $300 \mathrm{~nm}$ spot is due to the antennaluminescence, which is confirmed by the fact that for a retracted antenna one observes constant, uniformly distributed, $\mathrm{hBN}$-defect photon-emission, irrespective of the antennaposition, as shown in Figure $3 \mathrm{~d}$. The two darker spots are a)

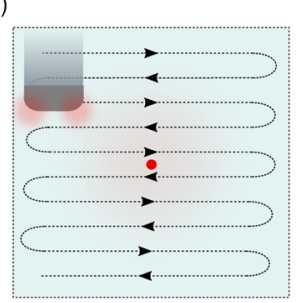

b)

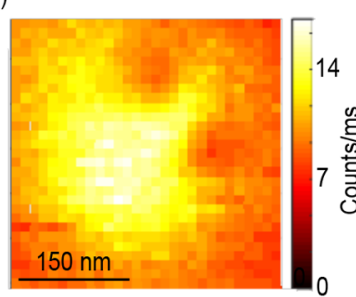

c)

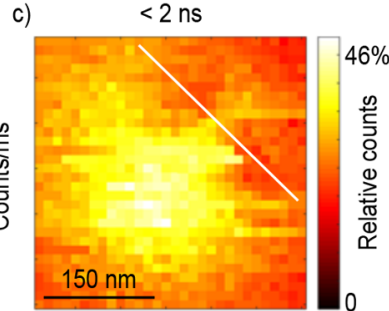

$2 \mathrm{~ns}$

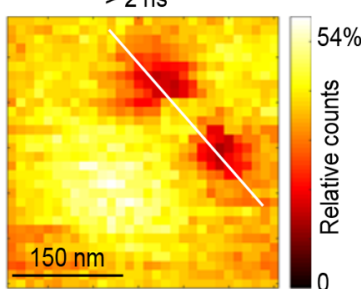

d)

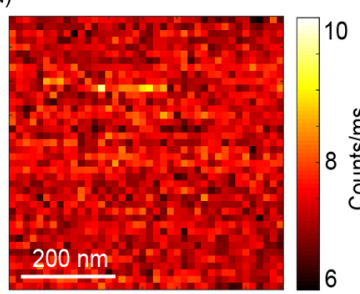

e)

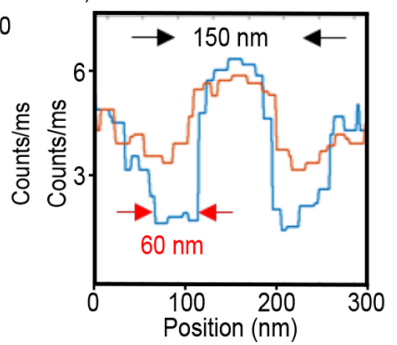

f)

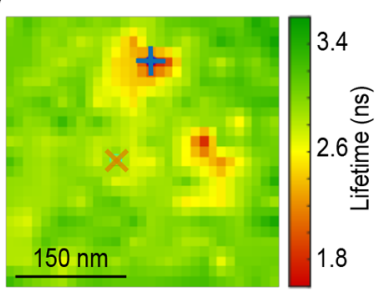

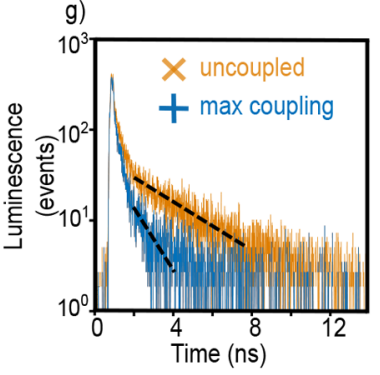

Figure 3. Nanoantenna mapping of individual hBN emission centers. (a) Schematic of a typical antenna probe scan over a continuously illuminated emitter. (b) Near-field image of an hBN defect using the flat dipolar antenna. (c) Time-gated luminescence maps using time intervals of 0-2 ns (left) and 2-25 ns (right). (d) Near-field image of an hBN-defect with the dipolar antenna probe retracted. (e) Line profiles along the paths indicated in (c) for intervals $0-2$ ns (orange) and $2-25 \mathrm{~ns}$ (blue). (f) Fluorescence lifetime-map obtained by fitting the $2-25 \mathrm{~ns}$ time-interval to a single exponential decay. (g) Photon-arrival histograms for the two points indicated in (f) alongside single-exponential fits (black dashed) indicating coupling-induced lifetime reduction. 
indicative of antenna hotspots, localized at the apexes of the antenna, where coupling is maximized. To quantify the analysis, we exploit the previously mentioned time-gating to separate the fast antenna luminescence $(<2 \mathrm{~ns})$ from the slower hBN emission. We detect all photons in a time-tagged fashion, which allows the generation of arrival-time filtered images during postacquisition analysis. ${ }^{24}$ Figure $3 \mathrm{c}$ shows the result of the time-gating analysis with the luminescence separated into two time-windows: $0-2$ ns and $2-25 \mathrm{~ns}$ intervals. The first image contains mainly antenna as well as some hBN-defect contributions and therefore strongly resembles the initial interaction-map presented in Figure 3b. The second image, however, almost exclusively contains hBNdefect luminescence, exhibiting a markedly enhanced doublespot contrast, whereas the antenna luminescence spot is almost fully suppressed leaving a near-constant background across the entire image. Figure $3 e$ further quantifies the effect of the 2 ns time-gating with 2 -fold contrast-improvement. The hBN-defect luminescence shows two depletion spots of $60 \mathrm{~nm}$ with maximum depletion of approximately $75 \%$ and separated by $150 \mathrm{~nm}$, which is in agreement with the aluminum antenna length of $160 \mathrm{~nm}$. We remark that these hotspot features are typical for either vertical or isotropic oriented emitters coupling to flat dipolar antennas as previously shown. ${ }^{23}$

Time-gating the hBN-signal ( $>2 \mathrm{~ns}$ ) facilitates the extraction of quantitative lifetimes under varying coupling-conditions, thus allowing generation of a full interaction lifetime map, as shown in Figure 3f. Here, the uncoupled hBN-defect exhibits a lifetime of about $3.5 \mathrm{~ns}$, which is in agreement with the value presented previously (Figure 1d). Once entering a hotspot, the lifetime decreases to approximately $1.6 \mathrm{~ns}$, that is, a reduction of 2.2 times (Figure $3 \mathrm{~g}$ ).

These observations are somewhat surprising, as the observed local antenna-defect coupling and lifetime reduction does not result in the anticipated luminescence enhancement. To concentrate the antenna-field further and allow a smaller separation between antenna-apex and hBN-defect, we performed the experiment presented above with a $30^{\circ}$ tilted dipolar antenna probe, which is excited out-of-resonance at $520 \mathrm{~nm}$ wavelength (SI Methods). The chosen tilted antenna configuration allows a tighter spatial light-confinement compared to the previously employed flat antenna probe, thus providing higher resolution of the $\mathrm{hBN}$-emission center and better coupling efficiency. Indeed, using the tilted antenna, we observe a single interaction-spot exhibiting a full width at half-maximum of only $45 \mathrm{~nm}$ (Figure $4 \mathrm{a}, \mathrm{b}$ ). The improved interaction-strength and light confinement of the tilted antenna-probe results in even more reduction in antennaluminescence. The antenna luminescence is slightly decreased with respect to the $\mathrm{hBN}$-defect emission, as the antenna is excited out-of-resonance, yet the tilted antenna is unable to increase the $\mathrm{hBN}$-emission-intensity beyond its free-space level.

As such, both the flat-dipolar as well as the tilted-dipolar antenna experiments presented in Figures 3 and 4 indicate that the antenna-hBN-defect interaction exclusively results in an overall emission depletion.

We have performed finite-difference time-domain (FDTD) simulations to explain our observations results. Figure 5 presents an overview of the simulation results for the flat dipole antenna. The calculation-geometry is outlined in Figure 5a. Here, the hBN-defect is located inside the hBN-flake at a depth of $5 \mathrm{~nm}$, whereas the antenna is located at a distance of $20 \mathrm{~nm}$ a)
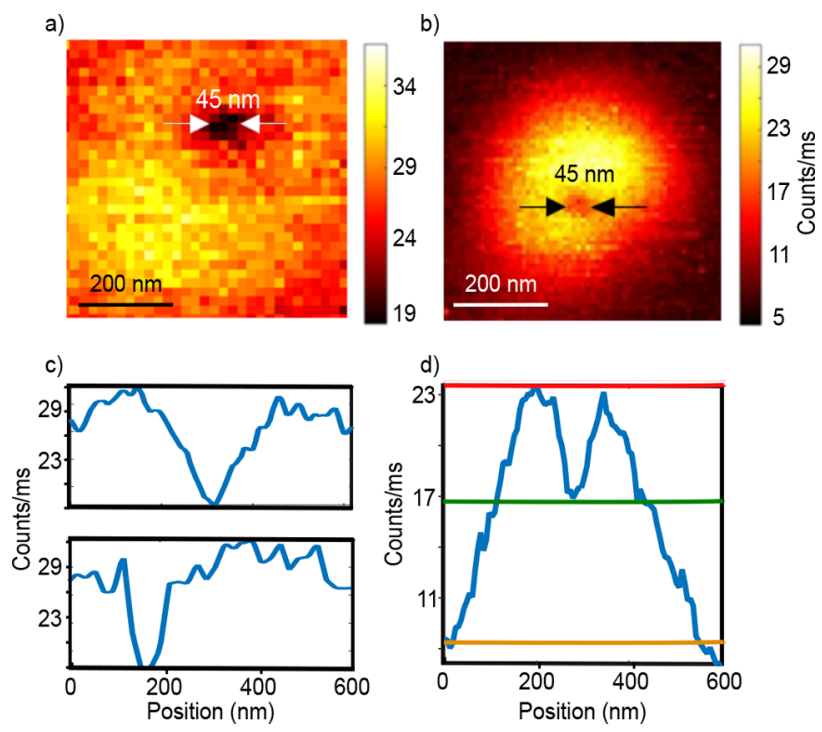

Figure 4. Imaging with a tilted dipolar antenna. (a) Probe scan image of an hBN emission center with the tilted dipolar antenna shown in Figure $2 \mathrm{~b}$. (b) Confocal fluorescence image of the same hBN defect with the antenna being positioned above it; the emitter is localized with a $45 \mathrm{~nm}$ size depleted spot. (c) Horizontal (top) and vertical (bottom) profiles obtained from the antenna-scan. (d) Horizontal profile of the confocal image.

from the hBN-surface, unless stated otherwise. The long resonant nanoantenna-axis is aligned with the $x$-direction. The calculated total electric field intensity in the vicinity of the antenna is shown in Figure 5b. Close to the surface, we observe an approximately 100-fold intensity increase with respect to the incoming field. However, due to the high refractive index of $\mathrm{hBN}(n=2.2)$ as well as the high radius of antenna-curvature of $25 \mathrm{~nm}$, the intensity enhancement inside the hBN-flake is only slightly above unity. ${ }^{25,26}$ As a consequence, the antennainduced excitation enhancement is approximately unity and, hence, negligible.

Figure $5 \mathrm{c}$ compares the calculated radiative and nonradiative rates alongside the luminescence lifetime for three different emission-dipole moment orientations. We concentrated on $x$-, $z$-, and isotropic-oriented emitters. We omitted the $y$-oriented case since it exhibits a characteristic four-lobe pattern ${ }^{23}$ which we did not observe in our measurements. We decided to include the isotropic case, as only $5 \%$ of all characterized hBNdefects showed antibunching, whereas the majority exhibited a very low DOP, suggesting that a large fraction of the $h B N$ defect emitters are composed of more than a single emitter. For all three cases, we observe a rate increase near the apex of the nanoantenna, both for the radiative as well as the nonradiative contribution, as expected for a resonant dipoleantenna. Assuming a luminescence lifetime of $3.5 \mathrm{~ns}$, the reduction is plotted in Figure 5c. A direct comparison with the experimentally observed lifetimes (Figure 3f) reveals a quantitatively good agreement with lifetime-minima separated by approximately $150 \mathrm{~nm}$. Yet assigning an emission-dipole orientation based on the results presented is rather difficult.

To further understand our observations, we simulated the emission quantum efficiency ( $Q E$ ) of the emitters, where we assume unity QE for uncoupled defects, based on previously reported literature values. ${ }^{27,28}$ As a result, upon interaction with the nanoantenna the QE cannot be improved by enhanced radiative rate and can only be reduced by enhanced 
a)

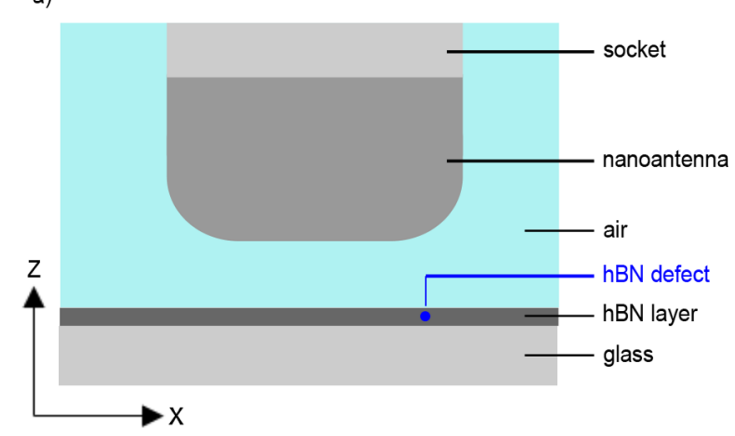

b)

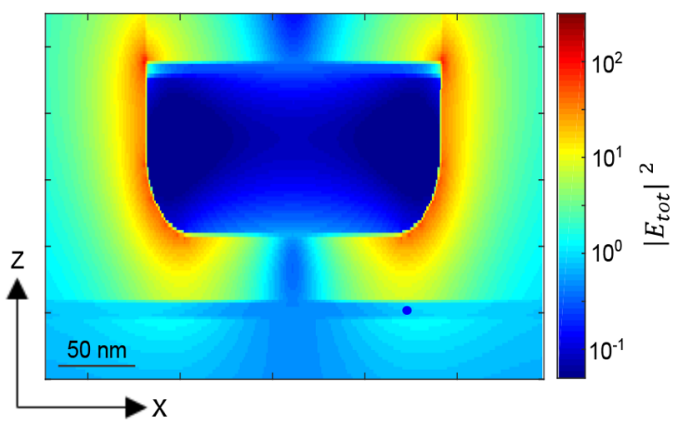

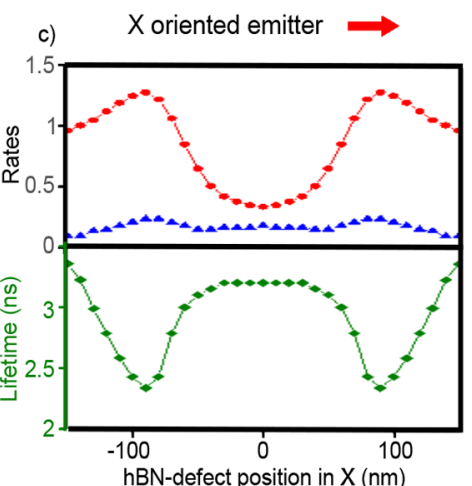

$\mathrm{hBN}$-defect position in $\mathrm{X}(\mathrm{nm})$

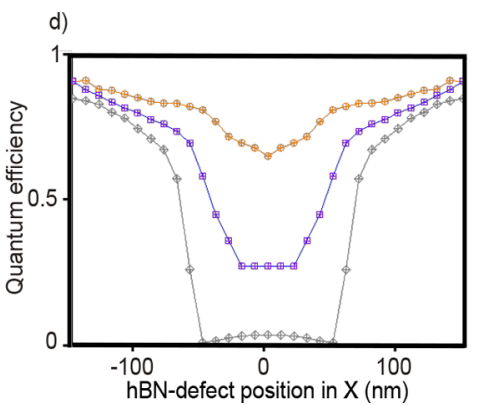

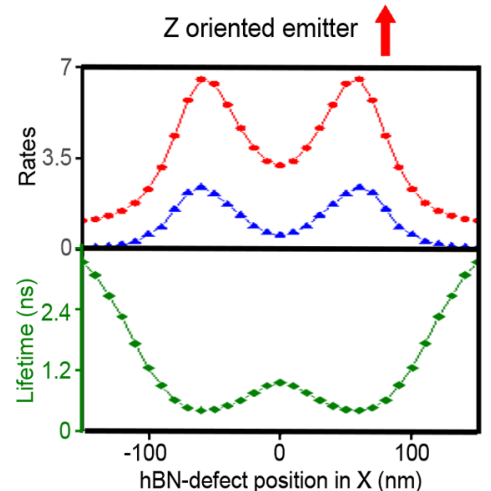

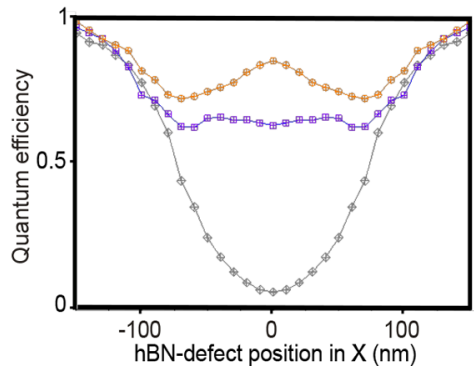

Isotropically oriented emitter
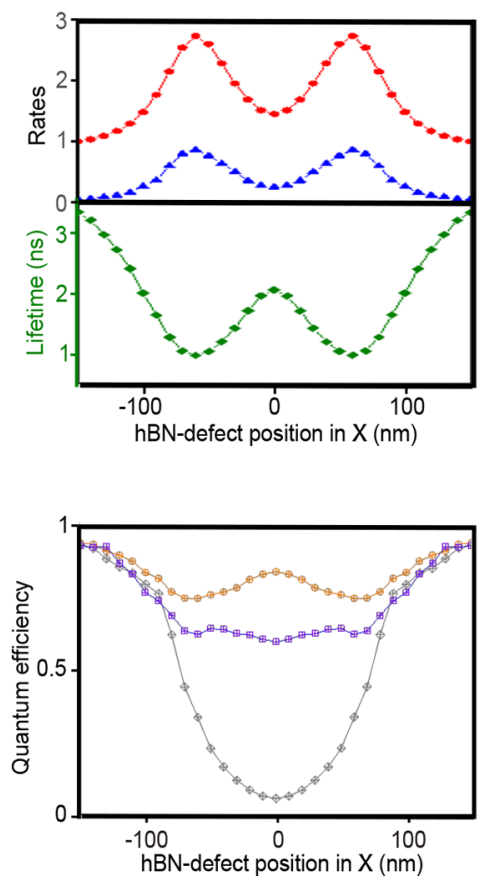

Figure 5. FDTD simulations of the excitation and emission properties of an hBN-defect located in close proximity to a dipolar nanoantenna. (a) Sketch of the typical antenna-hBN-defect emitter configuration. The plotted aspect ratio is elongated by a factor of 2 in the $z$-dimension for the sake of clarity. (b) Electric-field intensity map across the antenna when driven by a plane wave with a wavelength of $620 \mathrm{~nm}$. (c) Radiative rates (red) and nonradiative rates (blue), relative to the uncoupled emitter, as well as the luminescence (green), at an antenna-to-defect distance of 25 $\mathrm{nm}$, for $x$-oriented (left), $z$-oriented (center), and isotropic-oriented emitters (right). (d) Quantum efficiency of hBN emitters located at different $z$ distances of $25 \mathrm{~nm}$ (orange), $15 \mathrm{~nm}$ (purple), and $3 \mathrm{~nm}$ (gray) for $x$-oriented (left), $z$-oriented (center), and isotropic-oriented emitters (right).

nonradiative rate (Figure 5c). Figure 5d reports the resulting $\mathrm{QE}$ values for the nanoantenna located at 25,15 , and $3 \mathrm{~nm}$ above the hBN-surface, again for $x$-, $z$-, and isotropic-oriented emitters. Indeed, QE depends strongly on the antenna-emitter configuration. At a distance of $25 \mathrm{~nm}$, we observe predominantly radiative decay with limited losses, but already at a distance of $15 \mathrm{~nm}$ the nonradiative rate becomes nonnegligible and at $3 \mathrm{~nm}$ we observe pronounced quenching with the $\mathrm{QE}$ reaching values below $10 \%$.

On the basis of the simulations presented in Figure $5 c$,d, we re-examine the experimental results (Figure 3). First, the observed lifetime reduction shows quantitatively good agreement with the lifetime-simulations and does confirm considerable hBN-defect-antenna coupling. The simulations indicate that the excitation enhancement is strongly screenedoff due to the high-refractive index of $\mathrm{hBN}$, therefore we concentrate our analysis on the emission intensities (Figure 5d). In silico, $x$-oriented emitters exhibit emission profiles with a minimum at the center of the antenna, which fails to reproduce our experimental double-spot observations. Instead, $z$ - and isotropic-oriented emitters do show two local minima, exhibiting a QE decrease of $30 \%$ and $20 \%$, respectively. The measured emission depletion is $50-75 \%$, depending on the time-gating. The antenna is modeled as a perfectly smooth, single crystalline, metallic object. In reality, SEM images and optical measurements show, depending on fabrication conditions, that a typical Al antenna is polycrystalline with $20-40 \mathrm{~nm}$ size grains ${ }^{29}$ which can explain the differences in emission-quenching.

Overall, the simulations suggest that the hBN-emitter is either $z$ - or isotropic-oriented as we observe a double-lobe pattern rather than a central spot, as expected for an $x$-oriented dipole. On the basis of these observations, we conclude that the hBN-defect must be isotropically oriented as the zorientation is unlikely given the in-plane excitation polarization. In other words, the $\mathrm{hBN}$-emission center measured in Figure 3 is most likely composed of more than a single emitter, 

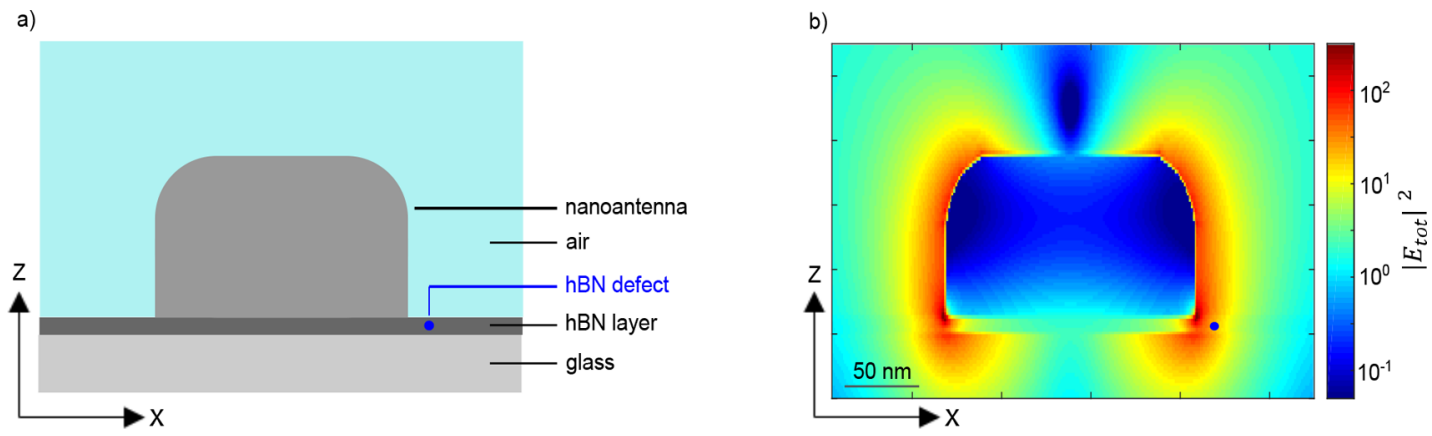

Figure 6. FDTD simulation of the excitation of an hBN defect in close proximity to a dipolar nanoantenna without air gap. (a) Sketch of a dipolar nanoantenna directly on top of a $5 \mathrm{~nm}$ thick hBN layer. The hBN emitter is located inside the hBN layer at a lateral distance of $5 \mathrm{~nm}$ from the antenna. (b) $x-z$ map of the electric field intensity around the structure shown in (a) excited at a wavelength of $620 \mathrm{~nm}$. The hBN-defect is indicated by the blue dot.

all of which are randomly oriented, thus forming an average isotropic emission-center.

The overall picture suggests that, in this specific configuration, luminescence cannot be enhanced by manipulating the emission properties. First, internal QE of the hBN-emitters is already near-unity. Second, the excitation enhancement is not sufficient to compensate for the antenna losses, which is a direct result of the high refractive index of hBN and the large index step to the air-gap between defect and antenna. As a result, the interaction between antenna and $\mathrm{hBN}$-defect leads to an overall emission-depletion, resulting in the observed dark spots at the antenna-apexes.

An important insight from our scanning antenna results is that one needs to avoid the low index air gap to gain $\mathrm{hBN}$ defect emission enhancement by antenna interaction. An alternative configuration is sketched in Figure 6a. Here, the aluminum antenna is fabricated directly on exfoliated hBN. Such a configuration can be realized fabricating the nanoantennas directly on the hBN-layer by e-beam lithography, whereas the $\mathrm{hBN}$-defect in close proximity might be induced by gallium FIB exposure, as reported recently in the literature. $^{30}$ The nanoantenna directly on the hBN-flake with sharp metal-hBN transition results in a local field intensity enhancement of 2 orders of magnitude inside the hBN-layer (Figure 6b). The strong excitation enhancement can compensate for the decrease of QE due to antenna losses. FDTD simulations of the total excitation-emission enhancement, for an $\mathrm{X}$ and isotropic oriented hBN-defect emitter embedded inside a $5 \mathrm{~nm}$ thick hBN-layer at $5 \mathrm{~nm}$ lateral distance from the antenna, show, respectively, $15 \times$ and $10 \times$ total enhancement of photon emission. The presented configuration design in Figure 6 enables an order of magnitude higher luminescence emission, that is, a brighter single-photon source. Importantly, the local excitation enhancement at the defect will make the defect emission dominate over the intrinsic antenna luminescence, allowing direct $\mathrm{hBN}$ photon emission detection, without the need of time-gating.

To summarize, for the first time we presented the experimental near-field coupling of a hBN-defect emitter to a tailor-made resonant plasmonic nanoantenna, which we systematically scanned through the $\mathrm{hBN}$-defect near-field, to generate a nanoscale interaction map. We employed dipolar nanorod antennas, both in planar and tilted geometry. The latter revealed single-hotspot images with $45 \mathrm{~nm}$ fwhm, indicating that individual $\mathrm{hBN}$-emission sites are confined to an area in size smaller than a few tenths of nanometers.
We quantified both the antenna-position-dependent luminescence intensity as well as the luminescence lifetime and furthermore implemented a time-gating strategy to separate the defect emission from the considerable intrinsic nanoantenna luminescence. Our data show that by relying on a resonant antenna configuration and the aforementioned timegating it is possible to localize $\mathrm{hBN}$-emission centers with nanometric accuracy within a $75 \%$ depletion dip while simultaneously shortening the lifetime by a factor of 2.2 .

The anticipated antenna enhancement was not observed in our near-field coupling experiments. The presented FDTD modeling suggests that emission enhancement is indeed difficult to achieve. This manifestation is due both to the noncoupled QE being close to unity and the fact that the high index hBN-layer serves as a screening layer, shielding the defect of the enhanced field in the vicinity of the nanoantenna. The latter point has important implications for plasmoniccoupling to solid-state emitters in general. Even though they are promising candidates for single-photon sources their small cross sections and resulting small emission rates call for efficient enhancement strategies to ensure competitive performance when compared to other promising alternatives such as single molecules. Here, plasmonic cavities, at first, might seem like a valid strategy but the large refractive index of typical materials such as TMDCs, silicon, or diamond prevents efficient electric field concentration. As such, it is necessary to reduce the materials' thickness to a minimum and to deterministically place the plasmonic antenna at a defined distance thus balancing excitation enhancement and quenching effects. At first, these constraints might appear prohibitive but the recent advances in nanofabrication based on He-based focused-ion-beam-milling and TMDC-manipulation put assembling such a coupled system within reach. Given that it is now possible to deterministically induce $\mathrm{hBN}$-defects at welldefined positions within an hBN-layer, ${ }^{30,31}$ we propose a topdown fabricated on-chip single-photon source, based on hBNdefects coupled to plasmonic nanoantennas, that allows an absolute luminescence enhancement of 10 - to 15 -fold, thus providing a one order-of-magnitude brighter single-photon source with no need for time-gating detection.

\section{ASSOCIATED CONTENT}

\section{Supporting Information}

The Supporting Information is available free of charge at https://pubs.acs.org/doi/10.1021/acs.nanolett.9b05268. 
Methods: preparation of hBN defects; antenna preparation; scanning antenna microscope; photon time-gating imaging and lifetime maps; second-order cross-correlation measurements; numerical simulations (PDF)

\section{AUTHOR INFORMATION}

\section{Corresponding Author}

Niek F. van Hulst - ICFO - Institut de Ciences Fotoniques, The Barcelona Institute of Science and Technology, Castelldefels, Barcelona 08860, Spain; ICREA, Institució Catalana de Recerca i Estudis Avançats, Barcelona 08010, Spain; (1) orcid.org/0000-0003-4630-1776

\section{Authors}

Nicola Palombo Blascetta - ICFO - Institut de Ciences Fotoniques, The Barcelona Institute of Science and Technology, Castelldefels, Barcelona 08860, Spain

Matz Liebel - ICFO - Institut de Ciences Fotoniques, The Barcelona Institute of Science and Technology, Castelldefels, Barcelona 08860, Spain

Xiaobo Lu - ICFO - Institut de Ciences Fotoniques, The Barcelona Institute of Science and Technology, Castelldefels, Barcelona 08860, Spain

Takashi Taniguchi - National Institute for Materials Science, Tsukuba, Ibaraki 305-0044, Japan; 이이.org/0000-00021467-3105

Kenji Watanabe - National Institute for Materials Science, Tsukuba, Ibaraki 305-0044, Japan; 이이이.0rg/000-00033701-8119

Dmitri K. Efetov - ICFO - Institut de Ciences Fotoniques, The Barcelona Institute of Science and Technology, Castelldefels,

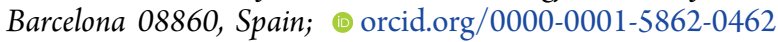

Complete contact information is available at: https://pubs.acs.org/10.1021/acs.nanolett.9b05268

\section{Author Contributions}

N.P.B., M.L., and N.F.v.H. designed the experiment. N.P.B. fabricated nanoantenna probes, performed the experiments, analyzed the data, and performed numerical modeling. T.T. and K.W. provided hBN. X.L. and D.K.E. exfoliated hBN flakes. N.P.B., M.L., D.K.E., and N.F.v.H. wrote the manuscript.

\section{Notes}

The authors declare no competing financial interest.

\section{ACKNOWLEDGMENTS}

N.F.v.H. acknowledges the financial support by the European Commission through ERC Advanced Grant 670949-LightNet. D.K.E. acknowledges support the H2020 Programme under Grant Agreement 820378, Project: 2D SIPC and the La Caixa Foundation. Authors acknowledge support by the Ministry of Science, Innovation \& Universities (RTI2018-099957-J-I00 and PGC2018-096875-B-I00), the Ministry of Economy (FIS2015-69258-P, BES-2016-078727, and "Severo Ochoa" program for Centers of Excellence in R\&D SEV-2015-0522), the Catalan AGAUR (2017SGR1369), Fundació Privada Cellex, Fundació Privada Mir-Puig, and Generalitat de Catalunya through the CERCA program. We thank Kevin Schädler for help in the initial phase of hBN sample fabrication.

\section{REFERENCES}

(1) Aspuru-Guzik, A.; Walther, P. Photonic quantum simulators. Nat. Phys. 2012, 8, 285-291.

(2) Lo, H.-K.; Curty, M.; Tamaki, K. Secure quantum key distribution. Nat. Photonics 2014, 8, 595-604.

(3) Reimer, M. E.; Bulgarini, G.; Akopian, N.; Hocevar, M.; Bavinck, M. B.; Verheijen, M. A.; Bakkers, E. P.A.M.; Kouwenhoven, L. P.; Zwiller, V. Bright single-photon sources in bottom-up tailored nanowires. Nat. Commun. 2012, 3, 737.

(4) Lounis, B.; Moerner, W. E. Single photons on demand from a single molecule at room temperature. Nature 2000, 407, 491-493.

(5) Lombardi, P.; Ovvyan, A. P.; Pazzagli, S.; Mazzamuto, G.; Kewes, G.; Neitzke, O.; Gruhler, N.; Benson, O.; Pernice, W. H. P.; Cataliotti, F. S.; Toninelli, C. Photostable Molecules on Chip: Integrated Sources of Nonclassical Light. ACS Photonics 2018, 5, 126-132.

(6) Doherty, M. W.; Manson, N. B.; Delaney, P.; Jelezko, F.; Wrachtrup, J.; Hollenberg, L. C.L. The nitrogen-vacancy colour centre in diamond. Phys. Rep. 2013, 528, 1-45.

(7) Hepp, C.; Muller, T.; Waselowski, V.; Becker, J. N.; Pingault, B.; Sternschulte, H.; Steinmuller-Nethl, D.; Gali, A.; Maze, J. R.; Atature, M.; Becher, C. Electronic structure of the silicon vacancy color center in diamond. Phys. Rev. Lett. 2014, 112, 036405.

(8) He, Y.; et al. Single quantum emitters in monolayer semiconductors. Nat. Nanotechnol. 2015, 10, 497-502.

(9) Siampour, H.; Kumar, S.; Bozhevolnyi, S. I. Chip-integrated plasmonic cavity-enhanced single nitrogen-vacancy center emission. Nanoscale 2017, 9, 17902-17908.

(10) Katsumi, R.; Ota, Y.; Osada, A.; Yamaguchi, T.; Tajiri, T.; Kakuda, M.; Iwamoto, S.; Akiyama, H.; Arakawa, Y. Quantum-dot single-photon source on a CMOS silicon photonic chip integrated using transfer printing. APL Photon. 2019, 4, 036105.

(11) Toninelli, C.; Early, K.; Bremi, J.; Renn, A.; Gotzinger, S.; Sandoghdar, V. Near-infrared single-photons from aligned molecules in ultrathin crystalline films at room temperature. Opt. Express 2010, $18,6577$.

(12) Hoang, T. B.; Akselrod, G. M.; Mikkelsen, M. H. Ultrafast Room-Temperature Single Photon Emission from Quantum Dots Coupled to Plasmonic Nanocavities. Nano Lett. 2016, 16, 270-275.

(13) Singh, A.; de Roque, P. M.; Calbris, G.; Hugall, J. T.; van Hulst, N. F. Nanoscale Mapping and Control of Antenna-Coupling Strength for Bright Single Photon Sources. Nano Lett. 2018, 18, 2538-2544.

(14) Tran, T. T.; Bray, K.; Ford, M. J.; Toth, M.; Aharonovich, I. Quantum emission from hexagonal boron nitride monolayers. Nat. Nanotechnol. 2016, 11, 37-41.

(15) Martinez, L. J.; Pelini, T.; Waselowski, V.; Maze, J. R.; Gil, B.; Cassabois, G.; Jacques, V. Efficient single photon emission from a high-purity hexagonal boron nitride crystal. Phys. Rev. B: Condens. Matter Mater. Phys. 2016, 94, 121405.

(16) Kianinia, M.; et al. Robust Solid-State Quantum System Operating at $800 \mathrm{~K}$. ACS Photonics 2017, 4, 768-773.

(17) Tran, T. T.; Wang, D.; Xu, Z.-Q.; Yang, A.; Toth, M.; Odom, T. W.; Aharonovich, I. Deterministic Coupling of Quantum Emitters in 2D Materials to Plasmonic Nanocavity Arrays. Nano Lett. 2017, 17, 2634-2639.

(18) Nguyen, M.; Kim, S.; Tran, T. T.; Xu, Z.-Q.; Kianinia, M.; Toth, M.; Aharonovich, I. Nanoassembly of quantum emitters in hexagonal boron nitride and gold nanospheres. Nanoscale 2018, 10, 2267.

(19) Xu, Z.-Q.; Elbadawi, C.; Tran, T. T.; Kianinia, M.; Li, X.; Liu, D.; Hoffman, T. B.; Nguyen, M.; Kim, S.; Edgar, J. H.; Wu, X.; Song, L.; Ali, S.; Ford, M.; Toth, M.; Aharonovich, I. Single photon emission from plasma treated $2 \mathrm{D}$ hexagonal boron nitride. Nanoscale 2018, 10, 7957-7965.

(20) Tran, T. T.; Elbadawi, C.; Totonjian, D.; Lobo, C. J.; Grosso, G.; Moon, H.; Englund, D. R.; Ford, M. J.; Aharonovich, I.; Toth, M. Robust Multicolor Single Photon Emission from Point Defects in Hexagonal Boron Nitride. ACS Nano 2016, 10, 7331-7338. 
(21) Tawfik, S. A.; Ali, S.; Fronzi, M.; Kianinia, M.; Tran, T. T.; Stampfl, C.; Aharonovich, I.; Toth, M.; Ford, M. J. First-principles investigation of quantum emission from hBN defects. Nanoscale 2017, 9, 13575-13582.

(22) Exarhos, A. L.; Hopper, D. A.; Grote, R. R.; Alkauskas, A.; Bassett, L. C. Optical Signatures of Quantum Emitters in Suspended Hexagonal Boron Nitride. ACS Nano 2017, 11, 3328-3336.

(23) Singh, A.; Calbris, G.; van Hulst, N. F. Vectorial nanoscale mapping of optical antenna fields by single molecule dipoles. Nano Lett. 2014, 14, 4715-4723.

(24) Wahl, M.; Rahn, H.-J.; Gregor, I.; Erdmann, R.; Enderlein, J. Dead-time optimized time-correlated photon counting instrument with synchronized, independent timing channels. Rev. Sci. Instrum. 2007, 78, 033106.

(25) Lee, S.-Y.; Jeong, T.-Y.; Jung, S.; Yee, K.-J. Refractive Index Dispersion of Hexagonal Boron Nitride in the Visible and NearInfrared. Phys. Status Solidi B 2019, 256, 1800417.

(26) Rah, Y.; Jin, Y.; Kim, S.; Yu, K. Optical analysis of the refractive index and birefringence of hexagonal boron nitride from the visible to near-infrared. Opt. Lett. 2019, 44, 3797.

(27) Schell, A. W.; Svedendahl, M.; Quidant, R. Quantum Emitters in Hexagonal Boron Nitride Have Spectrally Tunable Quantum Efficiency. Adv. Mater. 2018, 30, 1704237.

(28) Nikolay, N.; Mendelson, N.; Ozelci, E.; Sontheimer, B.; Bohm, F.; Kewes, G.; Toth, M.; Aharonovich, I.; Benson, O. Direct measurement of quantum efficiency of single-photon emitters in hexagonal boron nitride. Optica 2019, 6, 1084-1088.

(29) Albrecht, G.; Ubl, M.; Kaiser, S.; Giessen, H.; Hentschel, M. Comprehensive study of plasmonic materials in the visible and nearinfrared: Linear, refractory, and nonlinear optical properties. ACS Photonics 2018, 5, 1058-1067.

(30) Ziegler, J.; Klaiss, R.; Blaikie, A.; Miller, D.; Horowitz, V. R.; Aleman, B. J. Deterministic Quantum Emitter Formation in Hexagonal Boron Nitride via Controlled Edge Creation. Nano Lett. 2019, 19, 2121-2127.

(31) Choi, S.; Tran, T. T.; Elbadawi, C.; Lobo, C.; Wang, X.; Juodkazis, S.; Seniutinas, G.; Toth, M.; Aharonovich, I. Engineering and Localization of Quantum Emitters in Large Hexagonal Boron Nitride Layers. ACS Appl. Mater. Interfaces 2016, 8, 29642-29648. 